PESQUIMAT, Revista de la Fac.CC.MM. de la

UNIVERSIDAD NACIONAL MAYOR DE SAN MARCOS

Vol.IV, $N^{\circ} 2$, pág.1-6, LIMA-PERÚ. Diciembre 2001

\title{
DECAIMIENTO EXPONENCIAL PARA MATERIALES PARCIALMENTE TERMOESLÁTICO
}

\section{Alfonso Perez Salvatierra ${ }^{1}$}

ABSTRACT. En el presente trabajo se obtiene el decaimiento exponencial del sistema,

$$
\begin{gathered}
\mid \begin{array}{cl}
u_{t t}-\Delta u+\operatorname{div}(a(x) \nabla \theta)=0 \quad \text { en } \Omega \times\langle 0,+\infty\rangle \\
\theta_{t}-\triangle \theta+\operatorname{div}\left(a(x) \nabla u_{t}\right)=0 \quad \text { en } \Omega \times\langle 0,+\infty\rangle
\end{array} \\
\text { con las condiciones iniciales: } \\
\left(u_{0}, u_{1}, \theta_{1}\right) \in\left[H_{0}^{1}(\Omega) \cap H^{2}(\Omega)\right] \times\left[H_{0}^{1}(\Omega)\right] \times\left[H_{0}^{1}(\omega) \cap H^{2}(\omega)\right] \\
\text { donde } \omega \text { es una vecindad de una parte de } \partial \Omega=\Gamma
\end{gathered}
$$

\section{INTRODUCCIÓN}

Estudiamos el sistema parcialmente termoelástico $(*)$ con sus respectivas condiciones iniciales y de frontera

$(*)$

$$
\begin{aligned}
& \mid \begin{array}{ll}
u_{t t}-\Delta u+\operatorname{div}(a(x) \nabla \theta)=0 & \text { en } \Omega \times\langle 0,+\infty\rangle \\
\theta_{t}-\Delta \theta+\operatorname{div}\left(a(x) \nabla u_{t}\right)=0 & \text { en } \Omega \times\langle 0,+\infty\rangle
\end{array}
\end{aligned}
$$

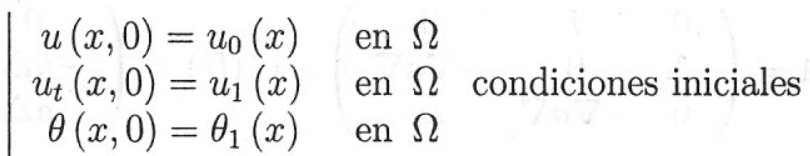

$$
\begin{aligned}
& u=0 \quad \text { sobre } \partial \Omega=\Gamma \\
& \theta=0 \quad \text { sobre } \partial \Omega=\Gamma \quad \text { condiciones de frontera }
\end{aligned}
$$

con las siguientes hipótesis; $\Omega \subseteq \mathbb{R}^{n}$, abierto bien regular, acotado, con frontera $\Gamma$ de clase $C^{2}, \Gamma=\Gamma_{1} \cup \Gamma_{2}: \Gamma_{1}=\{x \in \Gamma ; m(x) \cdot \nu>0\}, \Gamma_{2}=\{x \in \Gamma ; m(x) \cdot \nu<0\}$, $m(x)=x-x_{0}, \nu$ normal unitário, $x_{0} \in \mathbb{R}^{n}$ fijo. $a \in L^{\infty}(\Omega) \cap C^{2}(\Omega)$ tal que

\footnotetext{
${ }^{1}$ Universidad Nacional Mayor de San Marcos. Facultad de Ciencias Matemáticas.
} 
$a(x)>0$ en $\omega_{\varepsilon}$ y $a(x)=0$ en $\Omega-\omega_{2 \varepsilon}$ y satisface : $|\triangle a(x)|^{2} \leq C a(x),|\nabla a(x)|^{2} \leq$ $C a(x), \forall x \in \omega_{\varepsilon} ; \nabla \theta \cdot \nabla(\theta u) \geq 0$ en $\Omega$.

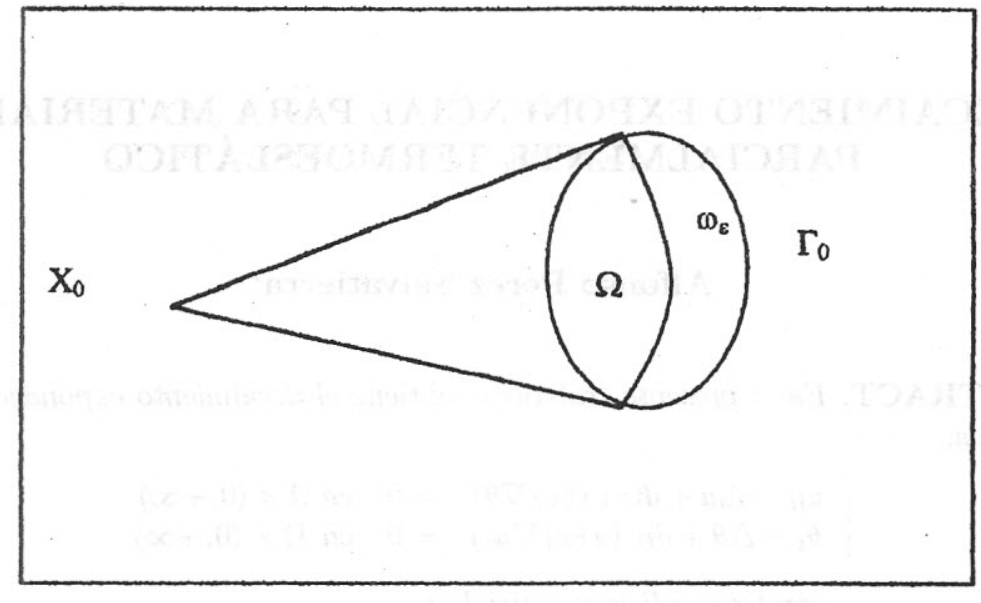

Figura 0.1

Además

$$
u_{0} \in H_{0}^{1}(\Omega) \cap H^{2}(\Omega), u_{1} \in H_{0}^{1}(\Omega), \theta_{0} \in H_{0}^{1}(\omega) \cap H^{2}(\omega) .
$$

Ahora haciendo $v=u_{t}$ en $(*)$ se obtiene el sistema matricial, con sus condiciones iniciales siguientes:

$$
\begin{aligned}
& U^{\prime}=A U+G(U), U_{0}=\left(\begin{array}{l}
u_{0} \\
u_{1} \\
\theta_{0}
\end{array}\right) \\
& U(0)=U_{0}
\end{aligned}
$$

donde $A: X \rightarrow X$ es un operador con las siguientes características:

$$
\begin{gathered}
A=\left(\begin{array}{ccc}
0 & I & 0 \\
\triangle & 0 & -\nabla a \nabla \\
0 & -\nabla a \nabla & \triangle
\end{array}\right), G(U)=\left(\begin{array}{c}
0 \\
-a \triangle \theta \\
-a \triangle v
\end{array}\right), U=\left(\begin{array}{l}
u \\
v \\
\theta
\end{array}\right) \\
D(A)=\left(H_{0}^{1}(\Omega) \cap H^{2}(\Omega)\right) \times H_{0}^{1}(\Omega) \times\left(H_{0}^{1}(\omega) \cap H^{2}(\omega)\right), \\
X=\overline{D(A)}=H_{0}^{1}(\Omega) \times L^{2}(\Omega) \times L^{2}(\omega) .
\end{gathered}
$$

Se define el producto interno en $X$ dado por

$$
(U, V) \int_{\Omega} \nabla u_{1} \nabla u_{2} d x+\int_{\Omega} v_{1} v_{2} d x+\int_{\Omega} \theta_{1} \theta_{2} d x
$$


con

$$
U=\left(\begin{array}{c}
u_{1} \\
v_{1} \\
\theta_{1}
\end{array}\right), \quad V=\left(\begin{array}{c}
u_{2} \\
v_{2} \\
\theta_{2}
\end{array}\right) \in X
$$

Luego aplicando el teorema de Lumer Philps y del hecho que $G(U)$ es localmente Lipschitziana, se deduce la existencia y la unicidad del sistema $(* *)$ y por ende de $(*)$. Para su decaimiento, por el método de Liapunov se define la energía

$$
E(t)=E_{1}(t)+E_{2}(t)
$$

donde

$$
\begin{aligned}
& E_{1}(t)=\frac{1}{2}\left[\left\|u_{t}(t)\right\|^{2}+\|\nabla u(t)\|^{2}+\|\theta(t)\|^{2}\right], \\
& E_{2}(t)=\frac{1}{2}\left[\left\|u_{t}(t)\right\|^{2}+\left\|\nabla u_{t}(t)\right\|^{2}+\|\theta(t)\|^{2}\right] .
\end{aligned}
$$

Definimos

$$
E_{3}(t)=\frac{1}{2}\left[\left\|\nabla u_{t}(t)\right\|^{2}+\|\Delta u(t)\|^{2}+\|\nabla \theta(t)\|^{2}\right]
$$

Multiplicando $(*)_{1}$ por $u_{t} \mathrm{y}(*)_{2}$ por $(\theta)$; luego sumando obtenemos:

$$
\frac{d}{d t} E_{1}(t)=-\|\nabla \theta(t)\|^{2}+2 \int_{\Omega} a(x) \nabla u_{t} \cdot \nabla \theta d x .
$$

Derivando $(*)_{1}$ respecto a $t$ y multiplicando por $u_{t t}$ además derivando $(*)_{2}$ respecto a $t$ y multiplicando por $\theta_{t}$, luego sumando obtenemos:

$$
\frac{d}{d t} E_{2}(t)=-\|\nabla \theta(t)\|^{2}+2 \int_{\Omega} a(x) \nabla u_{t t} \cdot \nabla \theta_{t} d x .
$$

Multiplicando $(*)_{1}$ por $-\triangle u_{t} \mathrm{y}(*)_{2}$ por $-\triangle \theta$; luego sumando obtenemos:

$$
\frac{d}{d t} E_{3}(t)=-\|\Delta \theta(t)\|^{2}-\int_{\Omega} \Delta a \nabla \theta \cdot \nabla u_{t} \cdot \nabla \theta_{t} d x .(x)+2 \int_{\Omega} a(x) \triangle \theta \triangle u_{t} d x .
$$

Se puede observar que en $E_{3}(t)$ aparece un término con derivada de segundo orden, el cual no es posible acotar usando las desigualdades de Sobolev. Para ello se hace uso de la siguiente proposición

\section{Proposición 1.}

Sea $v \in C\left(0, T ; H^{2}(\Omega)\right) \cap C^{1}\left(0, T ; H^{1}(\Omega)\right) \cap C^{2}\left(0, T ; L^{2}(\Omega)\right)$.

La solución de la ecuación 


$$
\begin{array}{rlrl}
v_{t t}-\Delta v & =f & & \text { en } Q \\
v=0 & & \text { sobre } \sum
\end{array}
$$

y $g=\left(g_{1}, \ldots, g_{n}\right) \in\left[C^{2}(\Omega)\right]^{n}$. Entonces $v$ satisface:

$$
\begin{gathered}
-\frac{d}{d t} \int_{\Omega} v_{t} g_{k} \frac{\partial v}{\partial x_{k}} d x= \\
-\int_{\Omega} f g_{k} \frac{\partial v}{\partial x_{k}} d x+\frac{1}{2} \int_{\Omega} \frac{\partial g_{k}}{\partial x_{k}}\left[\left|v_{t}\right|^{2}-|\nabla v|^{2}\right] d x+\int_{\Omega} \nabla v \cdot \nabla g_{k} \frac{\partial v}{\partial x_{k}} d x-\frac{1}{2} \int_{\Gamma} g_{k} v_{k}\left|\frac{\partial v}{\partial \nu}\right|^{2} d \Gamma .
\end{gathered}
$$

De donde se puede deducir para un $N$ bastante grande, el operador de Liapunov:

$$
\begin{gathered}
K(t)=N E(t)+E_{3}(t)+\int_{\Omega} a(x) \nabla \theta \cdot \nabla u_{t} d x \\
-C \int_{\Omega} a(x)^{2} u_{t} \Delta u d x-\varepsilon \int_{\Omega} a(x)^{3} \nabla u_{t} \nabla u_{t t} d x+2 \vec{\varepsilon} \int_{\Omega} \eta \nabla u_{t} \cdot \nabla u_{t t} d x, \\
N(t)= \\
\int_{\Omega}\left(a+a^{2}\right)\left[\left|\nabla u_{t}\right|^{2}+|\Delta u|^{2}\right] d x+\int_{\omega}\left[|\nabla \theta|^{2}+|\Delta \theta|^{2}\right] d x+\int_{\Gamma}\left|\frac{\partial \nabla u_{t}}{\partial \nu}\right|^{2} d \Gamma>0, \forall t>0 .
\end{gathered}
$$

Se prueba que:

$(* * *)$

$$
\frac{d}{d t} K(t) \leq-C N(t)-C_{0} \int_{\omega}\left|\nabla \theta_{t}\right|^{2} d x .
$$

Con las hipótesis de regularidad para $u, \theta$ y de $a(x)$ obtenemos:

\section{Proposición 2.}

$$
\begin{gathered}
\left(1-\frac{2 L}{T \sqrt{\beta}}\right) \int_{0}^{T} E(t) d t \leq C \int_{0}^{T} \int_{\Omega} a(x)\left(\left|\nabla u_{t}\right|^{2}+|\Delta u|^{2}\right) d x d t+ \\
C \int_{0}^{T} \int_{\omega}\left(|\nabla \theta|^{2}+\left|\triangle \theta_{t}\right|^{2}\right) d x d t+\int_{\Gamma}\left|\frac{\partial \nabla u_{t}}{\partial v}\right|^{2} d \Gamma>T>\frac{2 L}{\sqrt{\beta}} .
\end{gathered}
$$

Con las hipótesis de la proposición (2) obtenemos el teorema central. 


\section{Teorema}

Existen, $C \gamma<0$ tal que $E(t) \leq C E(0) \leq e^{-\gamma t}$.

\section{DEMOSTRACIÓN}

Es fácil ver: $\exists C_{0}, C_{1}>0$ tal que

(1)

$$
C_{0} E(t) \leq K(t) \leq C_{1} E(t) .
$$

De $(* * *)$ para $N$ bastante grande:

$$
\frac{d}{d t} K(t) \leq-C N(t)
$$

integrando de 0 a $T$ :

(2)

$$
K(t) \leq K(0)-C \int_{0}^{T} N(t) d t .
$$

De la proposición (2) y de la definición de $N(t)$ :

(3)

$$
\int_{0}^{T} E(t) d t \leq C \int_{0}^{T} N(t) d t
$$

Además de la definición de $N(t)$ y $(* * *)$ se tiene $\frac{d}{d t} K(t)<0, \forall t>0$, entonces $K(t)$ es decreciente; entonces,

(4)

$$
\int_{0}^{T} K(t) d t \geq T K(T)
$$

De (1), (2), (3) en (4)

obtenemos:

$$
\frac{T}{C_{1}} K(T) \leq \frac{1}{C_{1}} \int_{0}^{T} K(t) d t \leq \int_{0}^{T} E(t) d t \leq C \int_{0}^{T} N(t) d t \leq K(0)-K(T)
$$


Por lo tanto $\frac{T}{C_{1}} K(T) \leq K(0)-K(T)$,

$$
\left(1+\frac{T}{C_{1}}\right) K(T) \leq K(0) ; \alpha=1+\frac{T}{C_{1}}>1, K(T) \leq \alpha^{-1} K(0) .
$$

Luego, por la propiedad del semigrupo:

$$
K(t) \leq K(0) e^{-\gamma t}, \gamma=\frac{1}{T_{0}} \operatorname{Ln} \alpha>0, T_{0} \text { fijo. }
$$

Finalmente de (1):

es decir,

$$
E(t) \leq \frac{1}{C_{0}} K(0) e^{-\gamma t} \leq \frac{C_{1}}{C_{0}} E(0) e^{-\gamma t},
$$

$$
E(t) \leq C E(0) e^{-\gamma t}, \gamma=\frac{1}{T_{0}} \operatorname{Ln} \alpha .
$$

\section{CONCLUSIONES}

\section{Corolario.}

Bajo las condiciones del teorema si

$$
\left(u_{0}, u_{1}, \theta_{0}\right) \in H_{0}^{1}(\Omega) \times L^{2}(\Omega) \times L^{2}(\omega)
$$

entonces $\exists C>0, \gamma>0$ tal que $E_{1}(t) \leq C E_{1}(0) e^{-\gamma t}$.

\section{BIBLIOGRAFÍA}

[1] R. MUÑOZ, E. J. BISOGNIN, Exponential decay to partially thermoelastc materials. To appear.

[2] E. ZUAZUA, Exponential decay for the semilinear wave equation with locally distribuited damping. Comm PDE 15, (1990), p. 205-235.

[3] A. PEREZ SAlVATIERRA, Decaimiento de soluciones de ecuaciones Parcialmente Viscoelástico. Tesis Doctorado. 1993. Rio de Janeiro.

[4] R. J. MUÑOZ, Asymptotic behaviour in linear viscoelasticity. Quaterly on. Appl. Math., Vol.III,(1994), p.629-648.

[5] J. R. MUÑOZ, Global smooth solution and uniform rate of decay in nonlinear viscoelasticity. Reviews in Math. Phys., (1994), Vol 5,6. pp 855-868. 